\title{
A speed up of split-step wavelet for the computation of long range propagation
}

\author{
Thomas BONNAFONT*, Remi DOUVENOT*, Alexandre CHABORY*, \\ *ENAC, Université de Toulouse, Toulouse, FRANCE, thomas.bonnafont@enac.fr
}

\begin{abstract}
The atmospheric long-range propagation above the ground is of major importance for many ground systems as radars. The split-step wavelet method allows to compute efficiently this propagation using a pre-computed library for the wavelet-to-wavelet propagation. In this paper we propose a new method to efficiently compute the library needed for the propagation. From numerical experiments, we show that this novel method is faster to compute the library and as efficient in terms of precision and memory storage as the previous version.
\end{abstract}

Index Terms-split-step, wavelet, long range propagation.

\section{INTRODUCTION}

The long range propagation of electromagnetic waves is a topic of interest for many applications such as radar or navigation. One popular method to model this problem is splitstep Fourier (SSF) [1], which allows to take the refraction and the relief into account. The propagation is iteratively divided into two terms. One corresponds to the propagation in free space in the spectral domain, the second takes into account the refraction with a phase screen in the space domain.

Recently its discrete counterpart DSSF [2] has been proposed. Then a faster method has been developped: split-step wavelet (SSW) [2], [3]. The wavelet decomposition is used in place of the Fourier transform. This allows a faster computation of the propagation in large domains. This method uses the fast wavelet transform (FWT) of complexity $O(N)$, with $N$ the number of elements of the vector, whereas the FFT complexity is $O(N \log (N))$. Besides the wavelet decomposition allows a huge compression with a error due to thresholding that can be controlled.

The problem of SSW is the propagation which is more difficult and requires a pre-computed library of local propagators [6]. Since the time of computation of the library [3], [6] is not negligible compared to propagation time, we need to pre-compute it. Therefore, one limitation is that the steps of calculation must remain constant, preventing from using SSW in cylindrical coordinates for instance. In this paper we develop a new method using the local property of the wavelets to make the computation of this library negligible in time compared to the propagation, to improve the efficiency of SSW.

The problem is exposed in Section II. A brief reminder on wavelets is given in Section III. Then the SSW method is explained with the previous and new computations for the library in Section IV. We compare both methods. Finally a numerical test is presented with a comparison of both methods in Section V.

\section{CONFIGURATION}

In this article we compute the 2D forward propagation of the reduced field $u$ [1] over the ground with $y$ the direction of invariance. An $\exp (j \omega t)$ time dependence is assumed. At $x=0$ the field is known with a source at $x \leq 0$.

The domain is of size $x \in\left[0, x_{\max }\right]$ and $z \in\left[0, z_{\max }\right]$. First a discretization on the $z$ axis is made

$$
z_{p_{z}}=p_{z} \Delta z \text { with } p_{z} \in 0, \cdots, N_{z},
$$

with $N_{z}=\frac{z_{\max }}{\Delta z}$ the number of points. The field at each point is denoted $u\left[p_{z}\right]$. We also discretize on the $x$ axis with

$$
x_{p_{x}}=p_{x} \Delta x \text { with } p_{x} \in 0, \cdots, N_{x} .
$$

\section{BRIEF REMINDER ON WAVELETS}

The wavelet transform is a multilevel decomposition [5]. It is mostly used in signal theory and image processing, e.g. JPEG2000. Here we use it on the reduced field along $p_{z}$. A family of $L+1$ levels is used. The level $l=0$ corresponds to a non zero mean function, called the scaling function. It corresponds to the lower part of the spectrum as seen in Fig. 1. From levels $l=1$ to $l=L$ the functions are of zero mean and correspond to wavelets with different levels of dilation. Greater values of $l$ allow to take into account faster variations of the signal. The dilations allow to cover the spectrum as seen in Fig. 1. Translations allow to cover the spatial domain. Note that the support of the wavelet is a lot smaller than the spatial domain in practice.

Using the FWT [5] the field is decomposed as follows on $L+1$ levels

$$
u\left[p_{z}\right]=\sum_{p \in \mathbb{Z}} a_{L, p} \phi_{L, p}\left[p_{z}\right]+\sum_{l=1}^{L} \sum_{p \in \mathbb{Z}} d_{l, p} \psi_{l, p}\left[p_{z}\right] .
$$

The coefficients $a_{L, p}$ correspond to approximation coefficients, i.e. to the decomposition of the signal on the scaling function. The $d_{l, p}$ coefficients correspond to the details coefficients, ii.e. to the decomposition of the signal on each wavelet.

In this paper we use the symlet6, or "sym6", family which is of compact support and almost symmetric. The maximum level $L$ is chosen as $L=3$. These choices are discussed in [2]. 


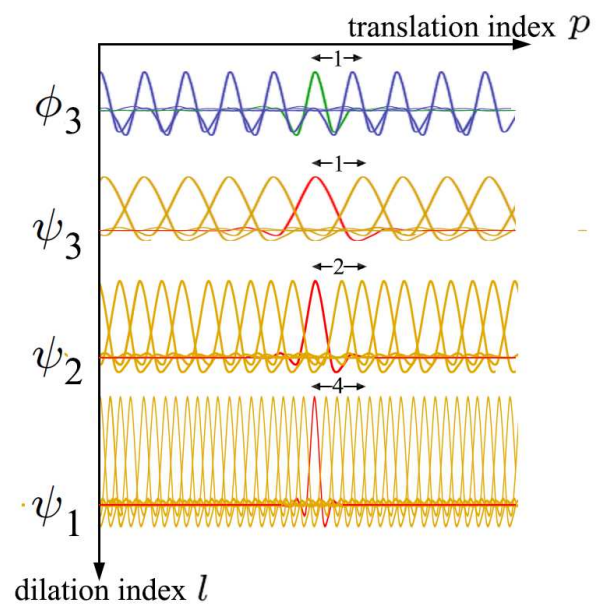

(a) Spatial domain.

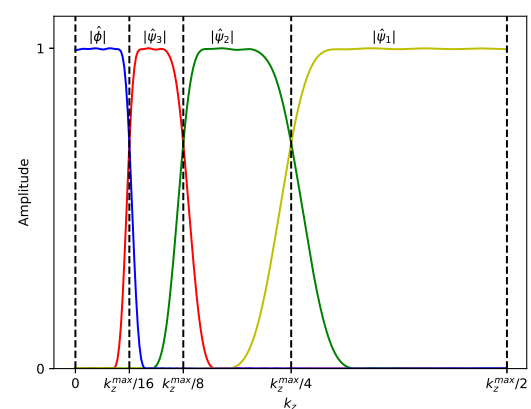

(b) Spectral domain.

Fig. 1: Wavelet basis.

\section{SPLiT-STEP WAVELET}

\section{A. Overview of split-step wavelet}

In this section an overview of the SSW method is given. In section IV-B and IV-C details are given on how to compute the library and the local propagation. SSW is an iterative method that follows the steps of SSF.

A step of SSW is performed as follows, with $u_{p_{x}}$ the field at position $x$ known

1) $u_{p_{x}}$ is decomposed in the wavelet domain using the FWT. We obtain $U_{p_{x}}$ a sparse vector of size $N_{z}$ containing the wavelet coefficients.

2) $U_{x}$ is propagated in the wavelet domain using a precomputed library. This library contains the waveletto-wavelet propagation for each level. We explain the method to compute the library in section IV-B. $U_{p_{x}+1}$ is obtained

3) $u_{p_{x}+1}^{\mathrm{fs}}$ is obtained using an inverse FWT on $U_{p_{x}+1}$. It corresponds to the propagation in free space.

4) The effects of both refraction and relief are taken into account in the space domain using a phase screen and translations to obtain $u_{p_{x}+1}$.

5) Apodisation using the Hanning window is performed.

This method is summed up in the block scheme of Fig. 2.
To compute efficiently the effect of the ground the local image theorem [2], [3] is used either directly for a PEC ground, or with the DMFT change of variable for an impedant ground. This method allows to compute the ground effects by adding a small number of points to the domain. The relief is considered using the staircase model.

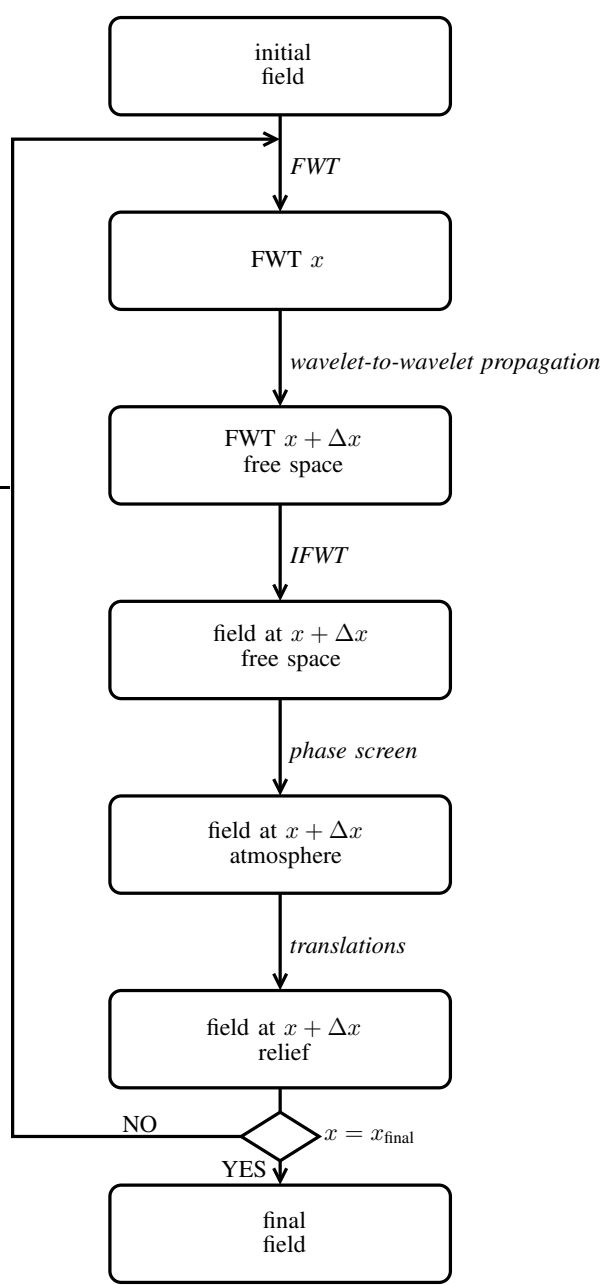

Fig. 2: Overview of SSW.

\section{B. Computing the library}

In this section, we first explain how to construct the library. Then the method we propose to improve this construction is detailed. Since the levels 0 and $L$ can be computed likewise, the level 0 is omitted here for conciseness.

To construct the library Algorithm 1 is used. First we need to compute the number of propagated wavelets needed at each level $l$

$$
q_{\max }(l)=2^{L+1-l} .
$$

Then each wavelet of level $l \in[1, L]$ and at position $q \in$ $\left[0, q_{\max }(l)[\right.$ is propagated using DSSF. To obtain the waveletto-wavelet propagation those propagated wavelets are decomposed using a FWT and stored in the library. A threshold $V_{m}$ is applied to ensure the sparsity of the propagator. Due to 
the invariance of the wavelet basis by translation, only one propagation is needed per level. Thus we only need $3 L+1$ DSSF. This method allows to have a fast computation of the library.

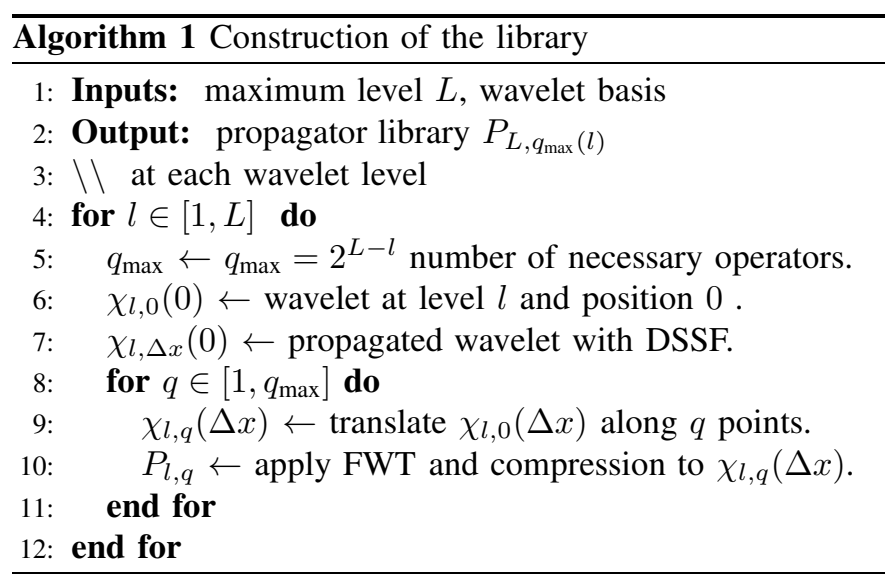

Using the compact and small support of the wavelets (compared to the size of the domain) we can obtain a faster method. Let be $N_{\text {supp }}(l)$, for $l \in[1, L]$, the number of points for the support of a wavelet, we have

$$
N_{\text {supp }}(l) \ll N_{z} \text { for } l \in[1, L] .
$$

So computing the DSSF only on the support of the wavelet is used to obtain a better method in term of time efficiency.

To do so, we introduce the support of the wavelet after propagation, $N_{\text {supp }}^{p}(l)$, at each level. To obtain this support we use the wide angle hypothesis and the support of the wavelet $N_{\text {supp }}(l)$ as shown in Fig. 3. We obtain the formula

$$
N_{\text {supp }}^{p}(l)=N_{\text {supp }}(l)+2 \frac{\Delta x \sin (\pi / 4)}{\Delta z} .
$$

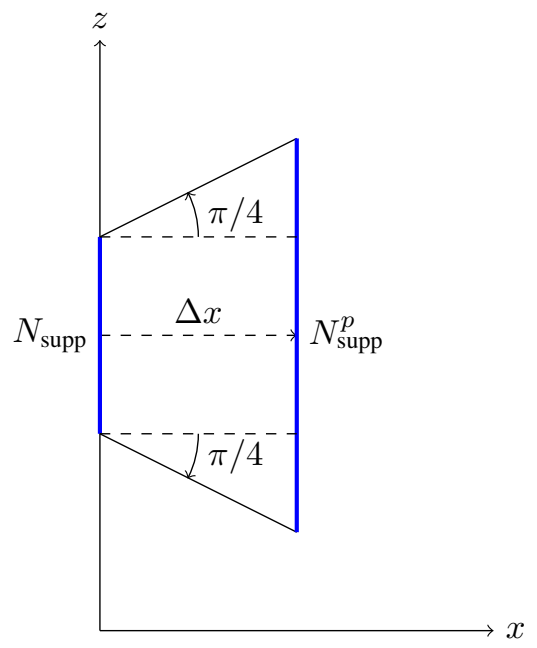

Fig. 3: Wavelet supports before and after propagation on one step.

To test this method, we compute the propagation using DSSF of a wavelet on the all domain, $N_{z}$ points, and only on $N_{\text {supp }}^{p}(l)$. We perform this test for several values of $N_{z}$. The wavelet is of level $l=1$, the steps $\Delta x$ and $\Delta z$ are $50 \mathrm{~m}$ and $0.5 \mathrm{~m}$, respectively. Fig. 4 shows the evolution of the computation time for DSSF for each case. Since $N_{\text {supp }}^{p}(l)$ is constant for a given level, the time of computation is constant as expected. An increase in the number of points yields a speed-up by a factor of 10 to 50 . For each level this improvement can be observed. Besides the normalised error between both methods is at maximum at $-250 \mathrm{~dB}$. This shows the interest of considering the support after propagation $N_{\text {supp }}^{p}(l)$.

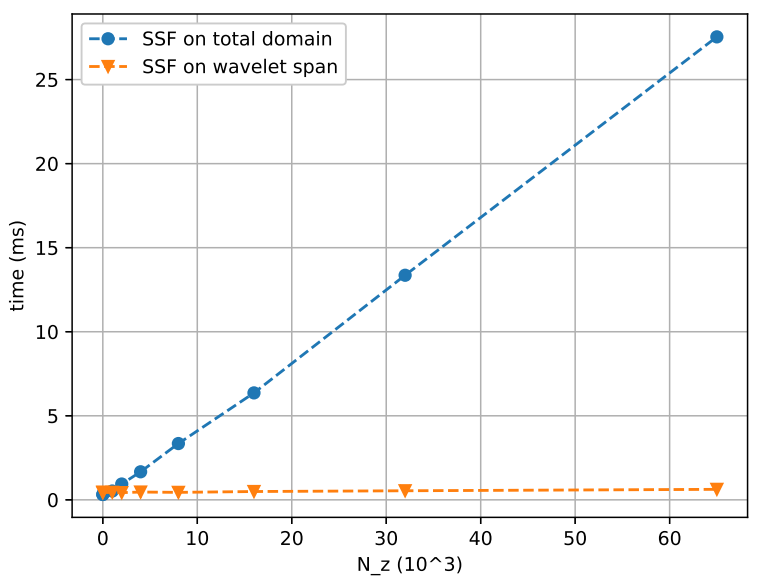

Fig. 4: Computation time of DSSF on the total domain and on $N_{\text {supp }}^{p}(l)$.

\section{Computing the propagation}

In this section, the method to propagate the field in the wavelet domain is explained.

Using the pre-computed library from IV-B, the propagation can be achieved. Using algorithm 2, all non-zero coefficients of the wavelet transform of the field $U_{x}$ are propagated using the appropriated propagator of the library. This propagator is chosen for a coefficient at position $p$ and level $l$ using

$$
q=p \quad\left(\bmod q_{\max }(l)\right) .
$$

Then each coefficient $U_{x}[l, p]$ is multiplied by the chosen operator to obtain elementary propagations. Finally all those elementary propagations are summed to obtain the total wavelet coefficients propagated $U_{x+\Delta x}$. The inverse FWT allows to come back to the space domain.

\section{NUMERICAL TEST}

In this section we compare the new version of SSW using the propagation computation on the wavelet span with the previous version of SSW.

The propagation of a complex source point (CSP) in a domain with a trilinear atmosphere and two triangular reliefs is computed. The CSP parameters are: a frequency $f=$ 

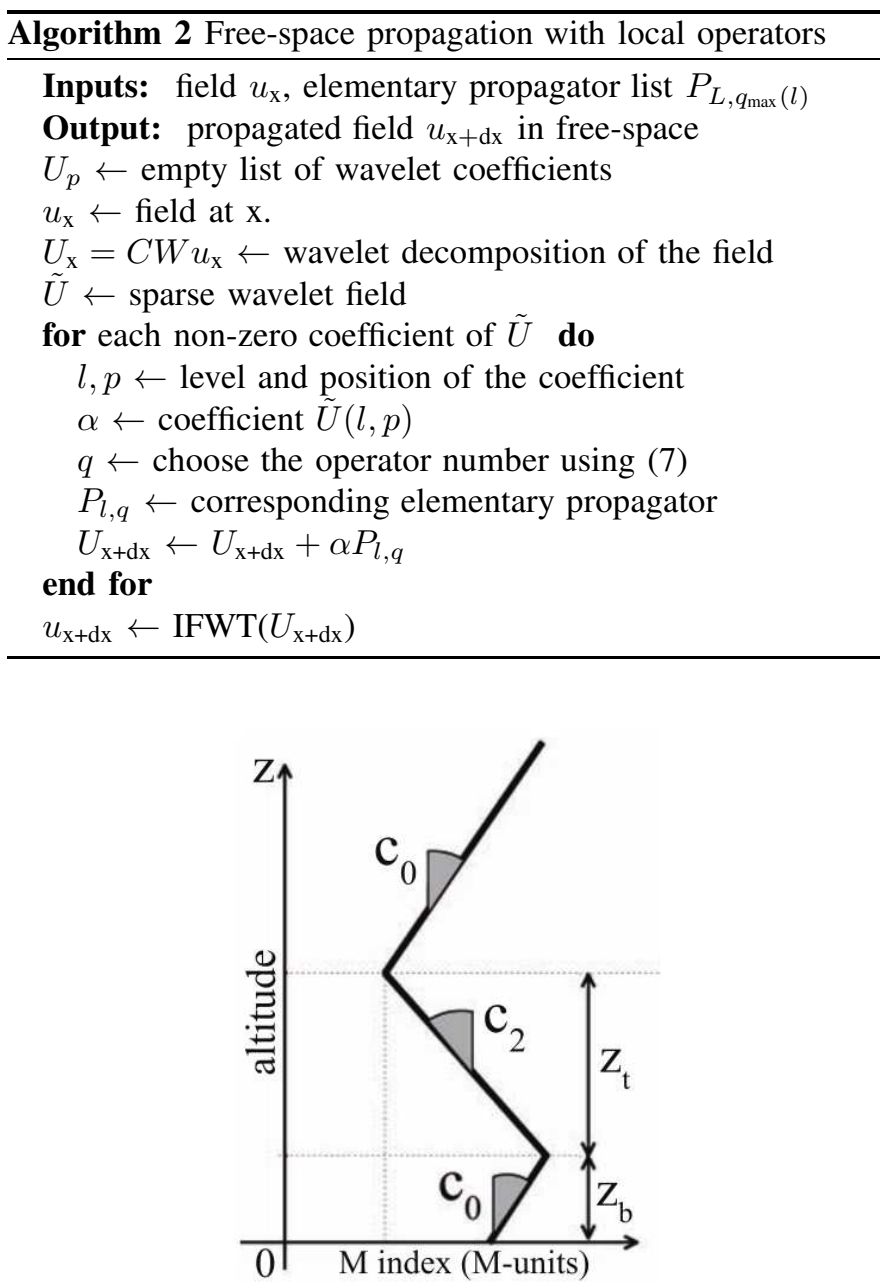

Fig. 5: Trilinear model of the atmosphere.

$300 \mathrm{MHz}$, with coordinates $x_{s}=x_{\mathrm{w} 0}+j k_{0} W_{0}^{2} / 2, z_{s}=30 \mathrm{~m}$, with $x_{\mathrm{w} 0}=-50 \mathrm{~m}$ and $W_{0}=5 \mathrm{~m}$.

We consider a refractive duct modelled by a trilinear atmosphere. The parameters for the refraction index are $M_{0}=$ 330 M-units, $z_{b}=100 \mathrm{~m}, z_{t}=200 \mathrm{~m}$, with gradients $c_{0}=0.118 \mathrm{M}$-units $/ \mathrm{m}$ and $c_{2}=-0.1 \mathrm{M}$-units $/ \mathrm{m}$, as illustrated in Fig. 5.

The relief is chosen as 2 small triangles of heights $100 \mathrm{~m}$ and $200 \mathrm{~m}$. The impedance ground is of parameters $\epsilon_{r}=20.0$ and $\sigma=0.02 \mathrm{~S} / \mathrm{m}$.

The domain is of size $x_{\max }=100 \mathrm{~km}$ in horizontal and $z_{\max }=2048 \mathrm{~m}$ in vertical. An apodization window of the vertical size is added. The grid size is $200 \mathrm{~m}$ in horizontal and $0.5 \mathrm{~m}$ in vertical.

The signal and propagator thresholds are $V_{s}=10^{-3}$ and $V_{m}=6 \times 10^{-5}$ respectively, so as to obtain an error of $-30 \mathrm{~dB}$ at the last iteration using the formula given by Zhou et al. [3], [2]. The image layer for the ground condition is of size $0.1 z_{\max }$.

The electric field using the local version of SSW is represented in Fig. 6. The effects of both the atmosphere and the relief (shadowing, reflection and diffraction) can be ob- served. The computation times and the memory usage of the methods are compared in Table I. First line corresponds to the computation time of the library. The second corresponds to the propagation time, which is the same for both methods. Then the total time and the memory needed for the library for both strategies is compared. We can see that the new method allows to be much faster to compute the library. Thus it could be computed at each iteration if necessary, in cylindrical coordinates for example.

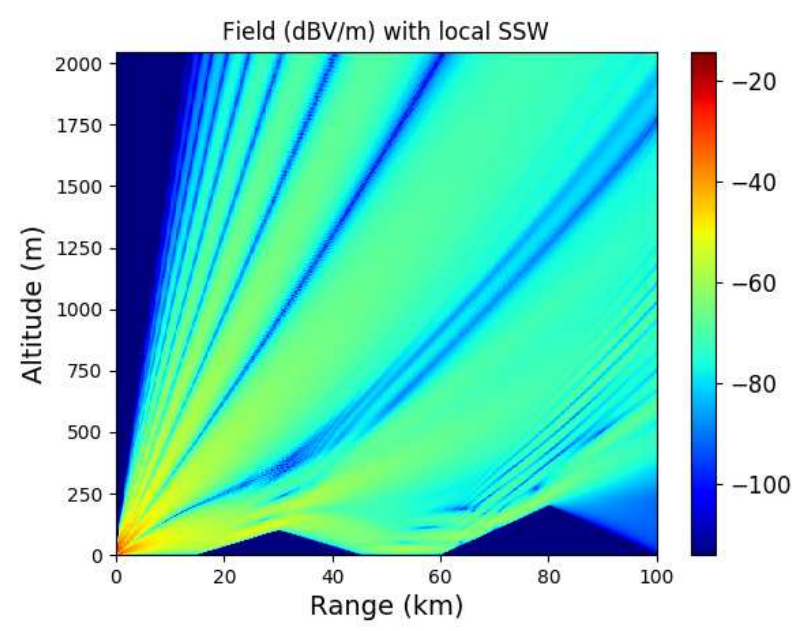

Fig. 6: Electric field (dBV/m) computed with SSW.

TABLE I: Time and memory storage needed for this scenario with each method.

\begin{tabular}{|l||c|c|}
\hline- & propagator on $N_{z}$ & propagators on $N_{\text {supp }}^{p}(l)$ \\
\hline Library (s) & 1 & 0.015 \\
\hline Propagation (s) & 7.1 & 7.1 \\
\hline Total (s) & 8.1 & 7.11 \\
\hline memory (Mb) & 2.1 & 2.1 \\
\hline
\end{tabular}

VI. CONCLUSION

In conclusion a new method to compute the library of propagators for SSW has been developed. This method is based on the small size of the support of the wavelets.

We have used the local property of the wavelets to improve the library computation. The new strategy allows to be 10 to 50 times faster for a comprehensive test case.

This strategy has been added to SSW. Results have shown that the method is as precise as the previous version of SSW. However the time efficiency is better with this new strategy.

Since the novel method computes the library in a time negligible compared to the propagation, we can compute it at each iteration. Thus it could be used with cylindrical coordinates where we need to compute the library at each step, or for adaptive step sizes.

\section{ACKNOWLEDGMENT}

The authors would like to thank the French Defense Agency (Direction Général de l'Armement, DGA) and the French Civil 
Aviation University (Ecole Nationale de l'Avitation Civile, ENAC) for the funding.

\section{REFERENCES}

[1] D. Dockery and J. R. Kuttler, " An improved impedance-boundary algorithm for Fourier split-step solutions of the parabolic wave equation," IEEE Transactions on Antennas and Propagation, 44, 1996, pp. 15921599, doi: 10.1109/8.546245.

[2] H. Zhou, " Modeling the atmospheric propagation of electromagnetic waves in 2D and 3D using Fourier and wavelet transforms," $\mathrm{PhD}$ thesis, Université de Toulouse, Université Toulouse III-Paul Sabatier, 2018.

[3] H. Zhou, A. Chabory, and R. Douvenot, " Modeling wave propagation by a split-step wavelet method," Journal of Computational Physics, in press.

[4] J. R. Kuttler and R. Janaswamy, " Improved Fourier transform methods for solving the parabolic wave equation," Radio Science, 37, no. 2, 2002. doi : $10.1029 / 2001$ RS002488

[5] S. Mallat, A Wavelet Tour of Signal Processing, Academic press, 1999.

[6] T. Bonnafont, R. Douvenot, and A. Chabory, " Split-step wavelet propagation modelling using local operators," 2019 URSI International Symposium on Electromagnetic Theory (EMTS), San Diego, 2019 